\title{
The Modeling of Gas-bubble Driven Circulations Systems
}

\section{O.J. ILEGBUSI and J. SZEKELY}

Department of Materials Science and Engineering, Massachusetts Institute of Technology, Cambridge, MA 02139, U.S.A.

(Received on October 30, 1990; accepted in the final form on May 18, 1990)

\begin{abstract}
A mathematical model is presented of the turbulent recirculating two-phase flow in gas-stirred systems. Conservation equations are solved for each phase variables and these are connected through interaction parameters. Allowance is made both for the direct influence of bubble transport on the turbulence field and the additional generation of turbulence energy at the liquid/gas interphase. In addition, the plume shape is a direct result of the computation rather than being prescribed a priori. Computed flowfields are presented for both air-water and nitrogen-steel systems. The air-water results compare well with established measurements of gas fraction and mean velocities. The steel system results seem to suggest that gas plumes in metals may be narrower than in water.
\end{abstract}

KEY WORDS: two-phase flow; gas-bubble circulation; air-water; nitrogen-steel; plume; modeling.

\section{Introduction}

Gas-bubble driven circulation systems play a very important role in many metals processing operations, including steelmaking, ladle metallurgy and the secondary refining of aluminum and copper.

Fluid flow problems associated with gas-plume driven circulation have received a great deal of attention over the years. ${ }^{1-6)}$ The initial efforts involved measurements using water models, which could then be scaled to represent molten metal systems. More recently mathematical models have been suggested, which are generally based on the numerical solution of the turbulent Navier-Stokes equations. ${ }^{6-14)}$ While considerable success has been achieved in using these models to represent experimental data, some quite serious unresolved problems still remain. There are two major causes that have to be considered.

(1) There are significant uncertainties in scaling from water to molten metals, principally due to the major difference in the interfacial tensions and the density ratios.

(2) A more fundamental problem in modeling is that in most of the work reported to date such as Refs. 5), 9) and 10), the gas-plume shape was assumed or postulated, rather than measured. Thus any predictive use of these models hinged critically on the assumptions made for the shape of the gas plume.

The critical importance of using the correct plume shape has been recently demonstrated by Wu et al ${ }^{12}$ and Sahajwalla et al. ${ }^{15)}$ where experimentally determined plume shapes (i.e., gas void fraction distribution) gave excellent agreement between the measured and the theoretically predicted velocity profiles.

Glearly in the development of a satisfactory, internally consistent mathematical model it would be highly desirable to be able to predict the plume shape from first principles, rather than having to rely on postulates. This real need is further amplified by the fact that the potentially most exciting application of these models lies in the area of direct ironmaking and novel secondary refining technologies, where the experience base is much more limited than in the more traditional processing systems, such as argon-stirred ladles.

The suggestion that gas-plume driven circulation systems may be represented on a fundamental basis is not new; indeed as early as 1981, Spalding ${ }^{16)}$ had suggested a computational methodology for tackling problems of this type. The principles involved are quite straight-forward: we have to write down separate conservation equations for the gas bubble and for the liquid phases and these are then connected through interaction coefficients. Indeed, several papers have been published during the past few years, describing this approach, notably by Gross et al. ${ }^{10)}$ and Schwartz et al. ${ }^{11}$

This previous work, while representing a promising start, is not quite complete; in the works by Schwartz et al. and Cross $e t$ al., only a partial allowance have been made for turbulence, while others have used constant, effective viscosity. A perhaps more serious practical problem has been the lack of systematic experimental verification of these predictions and the fact that the sensitivity of the computed results to the assumptions made for the intcraction coefficient has not been fully explored.

The present paper, the first in a series, is addressed to the resolution of these problems. In the approach to be taken full advantage will be made of the excellent detailed measurements recently reported by Castillejos and Brimacombe, ${ }^{17}$ ) Castillejos ${ }^{18}$ and of the PHOENIGS computational package. ${ }^{16)}$ In particular, we have allowed for both the direct influence of bubble transport on the turbulence field, and accounted for the additional generation of turbulence at the liquid/gas interphase.

The objective of the present paper is to develop a formulation of the plume driven circulation prob- 
lem and to present a critical comparison of the theoretical predictions with experimental measurementsfor a relatively simple physical configuration. Having gained a realistic perspective regarding the appropriateness of this approach, the subscquent papers will deal with multiple injection systems, deformed free surfaces and splashing.

\section{Formulation}

Let us consider a gas-plume driven circulation system, such as sketched in Fig. 1. It is seen that the rising gas plume will drag a liquid region, thus resulting in a recirculation flow pattern. In reality this gas plume will consist of gas bubbles dispersed in the liquid. Thus we seek to represent the behavior of the system by describing the coupled motion of the continuous liquid phase and the dispersed gas phase.

The following principal assumptions are made:

(1) The size of the gas bubbles is uniform throughout and the bubbles are introduced into the system, through a nozzle, at a given velocity.

(2) The free surface is assumed to be flat, but provision is made for the escape of the gas bubbles from the system.

(3) It is assumed that the $k-\varepsilon$ model may be applied to describe the behavior of the liquid phase.

(4) Transport between the gas phase and the liquid phase may be represented in terms of an interface-friction coefficient, the numerical value of which depends on the bubble size.

The remainder of the assumptions, such as steady state, no slip at the walls and axial symmetry are the usual ones made in modeling studies of this type.

Let us comment on the appropriateness of these assumptions.

The postulate of a uniform bubble size may seem a gross oversimplification; nonetheless, reasonable estimates of the mean bubble size may be made on the basis of turbulence theory. Furthermore, a parametric study will be conducted to explore the effect

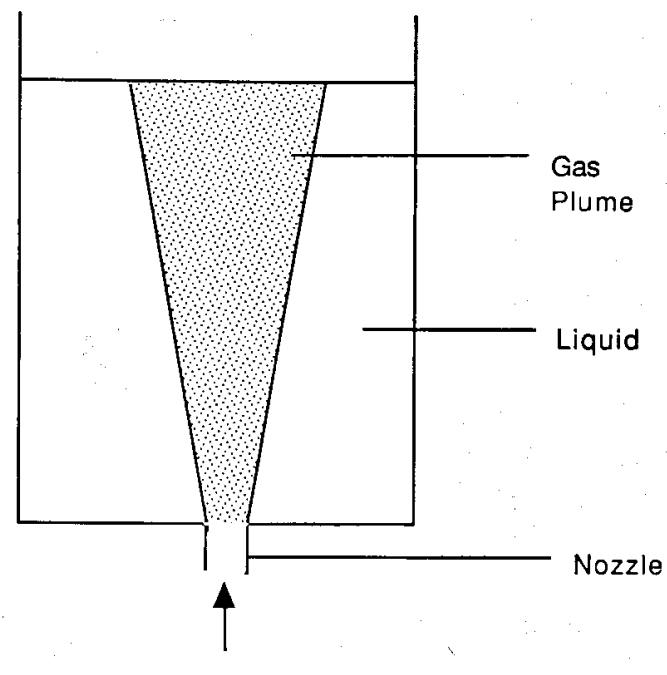

Gas

Fig. 1. A schematic sketch of a gas-plume circulation system. of changing the bubble size. Glearly, if desired and if so indicated by the experimental measurements, the model could be readily refined to allow for breakup or coalescence of the gas bubbles. In any case experience suggests that these processes that are responsible for breakup or bubble coalescence tend to take place predominantly in the vicinity of the injection nozzle.

The assumption of a flat, time independent free surface is usually made in studies of this type; nonetheless this is also an oversimplification, especially in the light of a recent paper by Sahajwalla et al. ${ }^{15}$ ) However, in the present work we have allowed for the escape of the gas in the vicinity of the free surface, thus minimizing the spurious radial flow that would occur as a result of this postulate. The assumption of a rigid flat surface will be relaxed in subsequent publications.

The assumption regarding the applicability of the $k-\varepsilon$ model to stituations of this type may also be an oversimplification, but one which had to be made in the absence of better alternatives available at present.

The interface-friction coefficient plays a pivotal role in the model. In the computed results to be presented subsequently we shall explore the effect of varying this parameter.

\subsection{Governing Equations}

Within the framework of the above assumptions, the governing equations may be put in the following forms:

\section{(1) Continuity Equation for Phase $j$}

$$
\nabla \cdot\left(\alpha_{j} \rho_{j} U_{j}-\rho_{j} D_{t} \nabla \alpha_{j}\right)=0
$$

where, $\alpha_{j}$ : the volume fraction for phase $j$

$\rho_{j}:$ the density

$U_{j}$ : the velocity.

$D_{t}$, the effective phase diffusion coefficient used to represent the random-motion flux associated with the interface between the phases is akin to the eddy viscosity and expressed as:

$$
D_{t}=\mu_{t} / \rho_{l}
$$

where, $\mu_{t}$ : the turbulent viscosity

$\rho_{l}:$ the liquid density.

(2) Conservation of Momentum for Generic Variable $\dot{\phi}_{j}$

$$
\nabla \cdot\left(\alpha_{j} \rho_{j} U_{j} \phi_{j}-\alpha_{j} L_{j} \nabla \phi_{j}\right)=S_{j}+S_{j}^{*}
$$

where, $\Gamma_{j}:$ an exchange coefficient representing the effects of turbulent and/or laminar diffusion within the phase

$S_{j}:$ the intra-phase source terms

$S_{j}^{*}:$ the inter-fluid source terms.

The appropriate forms of these terms are given in Table 1.

\section{(3) Total Conservation}

$$
\alpha_{g}+\alpha_{l}=1
$$

where, $\alpha_{g}, \alpha_{l}$ : gas fraction and liquid fraction, respectively. 
Table 1. Exchange coefficients and source terms.

\begin{tabular}{cccc}
\hline$\phi_{j}$ & $\Gamma_{j}$ & \multicolumn{1}{c}{$S_{j}$} & $S_{j}^{*}$ \\
\hline$U_{l}$ & $\mu_{\mathrm{eff}}$ & $-\alpha_{l} \frac{\partial P}{\partial x}+\alpha_{l} \rho_{l} g_{x}$ & $-F_{u}$ \\
$U_{g}$ & $\mu_{\mathrm{eff}}$ & $-\alpha_{g} \frac{\partial P}{\partial x}+\alpha_{g} \rho_{l} g_{x}$ & $F_{u}$ \\
$V_{l}$ & $\mu_{\mathrm{eff}}$ & $-\alpha_{l} \frac{\partial P}{\partial r}$ & $-F_{v}$ \\
$V_{g}$ & $\mu_{\mathrm{eff}}$ & $-\alpha_{g} \frac{\partial P}{\partial r}$ & $F_{v}$ \\
$k$ & $\frac{\mu_{t}}{\sigma_{k}}$ & $\alpha_{l} \rho_{l}(G-\varepsilon)+S_{k 1}+S_{k 2}$ & 0 \\
$\varepsilon$ & $\frac{\mu_{t}}{\sigma_{\varepsilon}}$ & $\alpha_{l} \rho_{l} \frac{\varepsilon}{k}\left(C_{1 \varepsilon} G-C_{2 \varepsilon} \varepsilon\right)+S_{\epsilon 1}+S_{\varepsilon 2}$ & 0 \\
\hline
\end{tabular}

\subsection{Constitutive Relations}

\section{(1) Turbulence Model}

The effective viscosity $\mu_{\mathrm{eff}}$ in Table 1 is represented with the $k-\varepsilon$ turbulence model which is applied to the liquid phase from the following expressions:

$$
\mu_{\mathrm{eff}}=\mu_{t}+\mu_{t}
$$

where $\mu_{t}$, the turbulent component is calculated from:

$$
\mu_{t}=C \mu \rho_{l} k^{2} / \varepsilon
$$

and $k$ and $\varepsilon$ are in turn obtained from transport equations similar to Eq. (2) with the corresponding exchange coefficients and source terms given in Table 1. In these expressions, $G$ is the volumetric rate of generation of $k$ which can be expressed as:

$$
G=\mu_{t}\left\{\left(\frac{\partial U_{l}}{\partial r}+\frac{\partial V_{l}}{\partial x}\right)^{2}+2\left[\left(\frac{\partial U_{l}}{\partial x}\right)^{2}+\left(\frac{\partial V_{l}}{\partial r}\right)^{2}\right]\right\}
$$

The second and third terms in the expressions for $S_{k}$ and $S_{c}$ are the novel aspects of the present formulation in the modeling of two-phase flow. $S_{k 1}$ and $S_{\varepsilon 1}$ are used to account for additional production terms at the liquid/gas interphase due to the sharp discontinuity and steep gradients of flow properties at such interface. These terms can be expressed as:

$$
\begin{aligned}
& S_{k 1}=C_{k 1} \rho_{l} \alpha_{g} \alpha_{l} G \\
& S_{\varepsilon 1}=C_{\varepsilon 1_{1} \rho_{l}} \alpha_{\eta} \alpha_{l} G \varepsilon / k
\end{aligned}
$$

The terms were first employed by Malin ${ }^{19)}$ to obtain correct spreading rates for jets and wakes with a twofluid model of turbulence. The author has also determined the values of the empirical coefficients $C_{k 1}$ and $C_{61}$ as given in Table 2 .

The last terms $S_{k 2}$ and $S_{62}$ are used to represent source terms associated with the migration of gas bubbles through the liquid and are expressed as:

$$
\begin{aligned}
& S_{k 2}=C_{k 2} \alpha_{g} o_{l} C_{f} \quad \ldots \\
& S_{\varepsilon 2}=C_{\varepsilon 2} \alpha_{g} \rho_{l} C_{f} \cdot \varepsilon / k
\end{aligned}
$$

where, $C_{f}$ : the interface friction coefficient given in the following section.
Table 2. Empirical constants used in the computation.

\begin{tabular}{ccccccccc}
\hline$C_{\mu}$ & $\sigma_{k}$ & $\sigma_{\varepsilon}$ & $C_{1 \varepsilon}$ & $C_{2 \varepsilon}$ & $C_{k 1}$ & $C_{k 2}$ & $C_{\varepsilon 1}$ & $C_{\mathrm{s} 2}$ \\
\hline 0.09 & 1.0 & 1.3 & 1.44 & 1.92 & 2.5 & 0.5 & 2.5 & 0.5 \\
\hline
\end{tabular}

Details of the derivation of Eqs. (10) and (11) are contained in a recent $\mathrm{PhD}$ thesis of Ref. 20) in which the values of the empirical constants $C_{k 2}$ and $C_{82}$ are also determined as given in Table 2.

\section{(2) Interphase Friction Forces}

The friction forces per unit volume that the liquid exerts upon the gas at the interface in the axial and radial directions are given respectively as:

$$
\begin{aligned}
& F_{u}=C_{f} \alpha_{g} \alpha_{l} \rho_{l}\left(U_{l}-U_{g}\right) \\
& F_{v}=C_{f} \alpha_{g} \alpha_{l} \rho_{l}\left(V_{l}-V_{g}\right)
\end{aligned}
$$

The implication of the above friction terms is that momentum is imparted to the slower-moving phase at the expense of the fast-moving phase. The friction coefficient $C_{f}$ is expressed as:

$$
C_{f}=\left(3 / 4 d_{b}\right) C_{d}\left|\mathbf{V}_{\mathbf{r}}\right|
$$

where $\left|\mathbf{V}_{\mathbf{r}}\right|$ is the absolute value of the relative velocity, and $C_{d}$ is the drag coefficient given by the following expression due to Clift $e t$ al. $^{21)}$ thus:

$$
C_{d}=\frac{24}{R e_{b}}\left(1+0.15 R e_{b}^{0.687}\right)+0.42 /\left(1+\frac{4.25 \times 10^{4}}{R e_{b}^{1.16}}-\right)
$$

In Eq. (14), $R e_{b}$ is the Reynolds number based on the gas bubble diameter $d_{b}$ and is defined as:

$$
R e_{b}=\frac{\rho_{l}\left|\mathbf{V}_{\mathbf{r}}\right| d_{b}}{\mu_{l}}
$$

The bubble diameter is in turn deduced from the relation established by Sano et al. ${ }^{22)}$ from energy balance considerations, thus:

$$
d_{b}=0.091\left(\frac{\gamma}{\rho_{l}}\right)^{0.5} U_{g}^{0.44}
$$

where, $r$ : the interfacial tension between the two phases.

It should be noted that Eq. (17) is strictly applicable to a bubble column of specified diameter. We have assumed that this expression may be applicable to the present system.

\subsection{Boundary Conditions}

The conditions employed at the various computational boundaries are as follows:

(1) Inlet Nozzle

$$
\begin{aligned}
& V_{l}=V_{g}=U_{l}=0 \\
& U_{g}=\frac{4 Q}{\pi d_{0}^{2}}
\end{aligned}
$$

where, $Q$ : the gas flow rate

$d_{0}:$ the nozzle diameter.

This implies that only a vertical gas velocity exists at 
the inlet nozzle.

\section{(2) Solid Walls}

At the walls, all velocities are set to zero. However, in order to preclude solution of the governing equations right to solid boundaries where steep gradients of flow variables occur, a log-law wall function is employed to deduce $k$, $\varepsilon$, wall shear stress and velocity components parallel to the boundary at the first computational grid point adjacent to this boundary. Details of this approach due to Launder and Spalding ${ }^{23)}$ are well documented and will not be repeated here.

\section{(3) Free Surface}

The free surface is assumed to be flat, frictionless and impervious to liquid. However, gas is allowed to leave at the rate at which it arrives at the surface. These conditions are represented mathematically as follows:

$$
\frac{\partial V_{l}}{\partial x}=\frac{\partial k}{\partial x}=\frac{\partial \varepsilon}{\partial x}=\frac{\partial U_{g}}{\partial x}=\frac{\partial \alpha_{g}}{\partial x}=0
$$

A reference pressure of zero is prescribed at a computational node at the surface.

\section{(4) Symmetry Axis}

A no-flux condition (zero normal gradients of flow variables) is imposed at the axis.

\section{Computational Details}

The set of equations detailed above are solved with the fully implicit finite domain numerical scheme embodied in the PHOENICS ${ }^{16}$ computational code. The solution procedure is based on the SIMPLE algorithm. ${ }^{24)}$

Systematic refinement shows that a non-uniform grid of 18 by 18 in the axial and radial directions, respectively, gives grid-independent and hence numerically accurate results. Typical results of the grid-refinement test are given in Table 3 where it is seen that the values of the gas fraction for case 1 at two axial locations do not change significantly beyond a grid of $18 \times 18$ points. This grid structure is employed for all the results to be presented here.

\section{Computed Results}

In the following we shall present a collection of the computed results in two groupings:

4.1. Water model systems, where detailed measurements are available, and

4.2. Nitrogen-stirred ladle systems, which are of practical interest to steelmakers.

Table 3. Grid refinement tests on the gas fraction at mid height.

\begin{tabular}{rrrrrrr}
\hline & $10 \times 15$ & $15 \times 15$ & $15 \times 18$ & $18 \times 18$ & $18 \times 24$ & $24 \times 24$ \\
\hline 10 & 0.332 & 0.345 & 0.352 & 0.355 & 0.356 & 0.3565 \\
30 & 0.171 & 0.178 & 0.185 & 0.188 & 0.189 & 0.1892 \\
\hline
\end{tabular}

\subsection{Water Model Systems}

4.1.1. The Measurements of Castillejos and Brimacombe ${ }^{17)}$

In a recent paper, Castillejos and Brimacombe ${ }^{17)}$ presented an extensive set of measurements describing the behavior of gas-plume driven circulation systems, using air and water. Indeed an even more comprehensive set of measurements is available in Castillejos. ${ }^{18)}$

In essence these measurements involved bubbling air through a cylindrical container, holding water, and measuring the void fraction distribution, for a variety of operating conditions. The principal system parameters are summarized in Table 4 and details of the specific cases are given in Table 5 .

Figs. 2 to 4 show a comparison between the theoretical predictions, developed in the present paper and the experimental measurements for the three cases considered. The broken line actually represents a correlation developed from the experimental measurements. The parameter appearing on the horizontal axis is the dimensionless axial distance, where $d_{0}$ is the inlet nozzle diameter for the gas. The parameter appearing on the ordinate is the gas fraction at the axis. Figs. 2 and 3 refer to two different gas flow rates, while Fig. 4 depicts a system with a different inlet nozzle diameter. The agreement between measurements and predictions is seen to be quite good for all three cases, especially close to the inlet nozzle. A better agreement between measurements and predictions could perhaps be achieved by refining the boundary conditions at the upper surface. This point will be explored in a subsequent publication.

The following three figures 5 to 7 address the radial (similarity) distribution of the void fraction, at a vertical position midway between the nozzle and the free surface. The parameter at the ordinate is the normalized gas fraction, with respect to its maximum value, while the abscissa is the dimensionless radial distance. Here the agreement is seen to be very good

Table 4. Principal input parameters used in the computation.

\begin{tabular}{lcll}
\hline \multicolumn{1}{c}{ Parameter } & Castillejos $^{17)}$ & $\begin{array}{c}\text { Grevet } \\
\text { et } \text { al. }^{5}\end{array}$ & $\begin{array}{c}\text { Steel } \\
\text { system }\end{array}$ \\
\hline $\begin{array}{l}\text { Diameter of vessel }(\mathrm{m}) \\
\text { Height of liquid in }\end{array}$ & 0.5 & 0.6 & 3.2 \\
$\quad$ vessel $(\mathrm{m})$ & 0.4 & 0.6 & 2.5 \\
Gas flow rate $(l / \mathrm{s})$ & $0.371-0.876$ & 0.217 & 43 \\
Nozzle diameter $(\mathrm{cm})$ & $0.41-0.635$ & 1.27 & 4 \\
Gas & Air & Air & Nitrogen \\
Liquid & Water & Water & Steel \\
\hline
\end{tabular}

Table 5. The specific cases considered for the experimental condition of Gastillejos. ${ }^{17}$

\begin{tabular}{cccc}
\hline Parameter & Case 1 & Case 2 & Case 3 \\
\hline Gas flow rate $\left(\mathrm{cm}^{3} / \mathrm{s}\right)$ & 371 & 876 & 876 \\
Nozzle diameter $(\mathrm{cm})$ & 0.635 & 0.635 & 0.410 \\
$\begin{array}{c}\text { Height of liquid in } \\
\text { vessel }(\mathrm{m})\end{array}$ & 0.4 & 0.4 & 0.4 \\
\hline
\end{tabular}




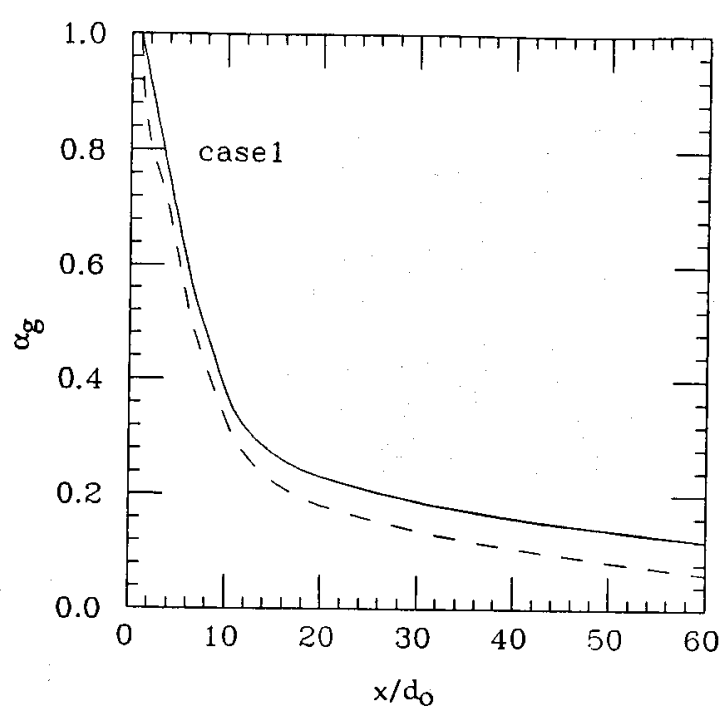

Fig. 2. Axial variation of gas fraction in a water model for the experimental conditions of Castillejos, ${ }^{17)}$ Case 1.

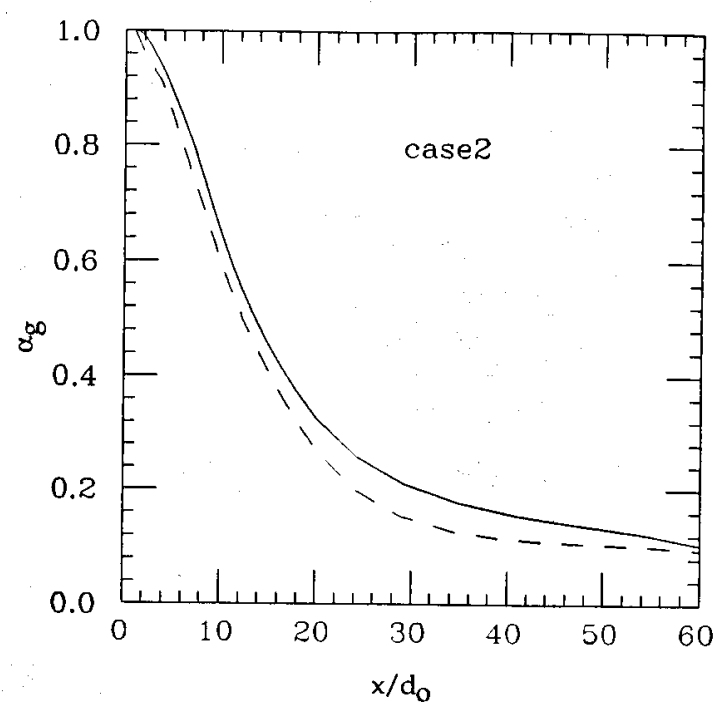

Fig. 3. Axial variation of gas fraction in a water model for the experimental conditions of Castillejos, ${ }^{17)}$ Case 2.

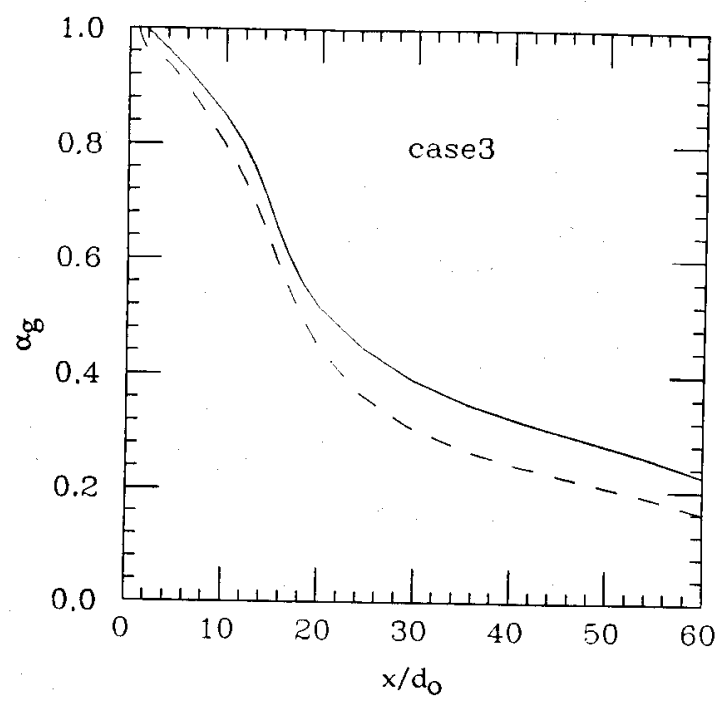

Fig. 4. Axial variation of gas fraction in a water model for the experimental conditions of Castillejos, ${ }^{17}$ Case 3.

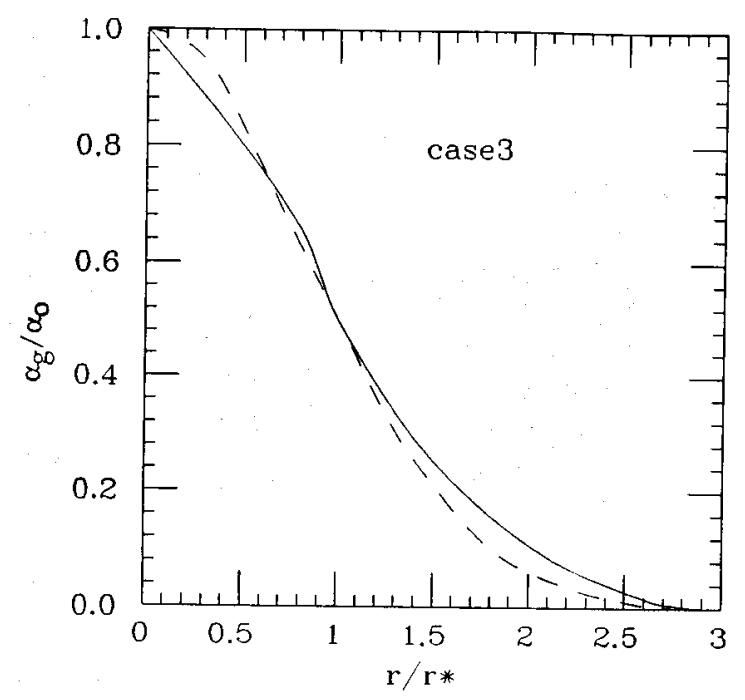

Fig. 5. Similarity profile of gas fraction in a water model for the experimental conditions of Castillejos, ${ }^{17)}$ Case 1.

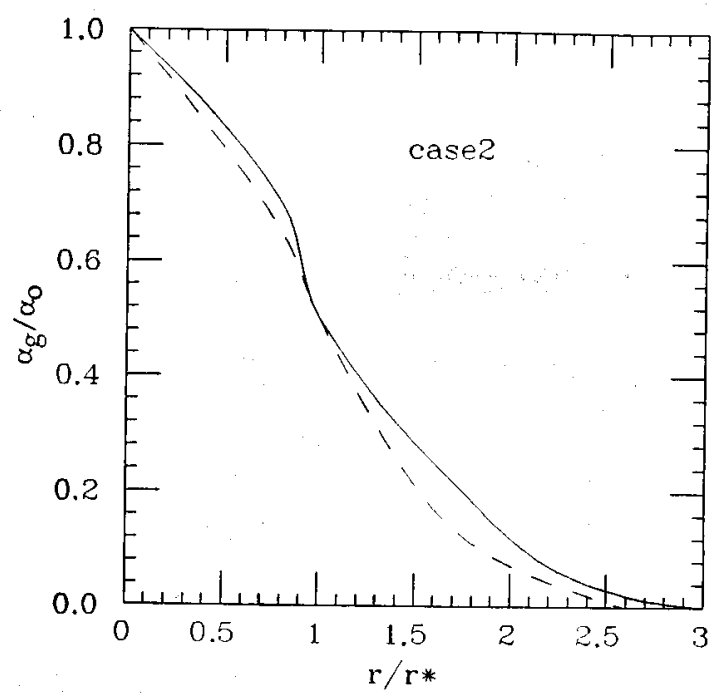

Fig. 6. Similarity profile of gas fraction in a water model for the experimental conditions of Castillejos, ${ }^{17)}$ Case 2.

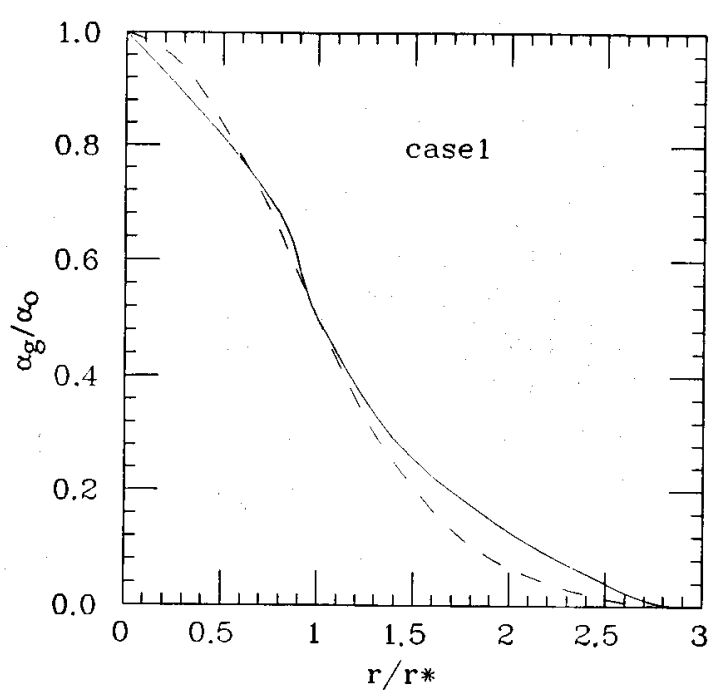

Fig. 7. Similarity profile of gas fraction in a water model for the experimental conditions of Castillejos, ${ }^{17}$ Case 3. 
between the measurements and the predictions.

\subsubsection{The Measurements of Grevet et al..$^{51}$}

Having obtained a reasonably satisfactory representation of the gas fraction, we may now proceed to calculate the velocity distribution in the system. Here the work of Grevet et al. ${ }^{5)}$ provides a good basis for comparison.

Fig. 8 shows the computed velocity distribution, for the experimental conditions reported by Grevet et al..$^{5)}$ while the following Figs. 9 and 10 show a comparison of the measurements with the predictions for the liquid velocity. The agreement between measurements and predictions appears to be excellent.

At this point it may be worthwhile to explore the sensitivity of the results to the numerical values chosen for the interface friction coefficient. This is done in Fig. 11 where it is seen that a twofold change in the interfacial friction coefficient does not appear to have a major effect on the mean velocity. The latter is calculated from the relation.

$$
U_{m}=\left|\alpha_{g} U_{g}+\alpha_{l} U_{l}\right|
$$

It is not immediately obvious whether the effect of $C_{f}$ on the gas fraction will be more pronounced than on the velocity.

It follows from the foregoing that the proposed representation does provide a reasonable means for modeling the behavior of gas plumes in water and that the computed predictions appear to be in good

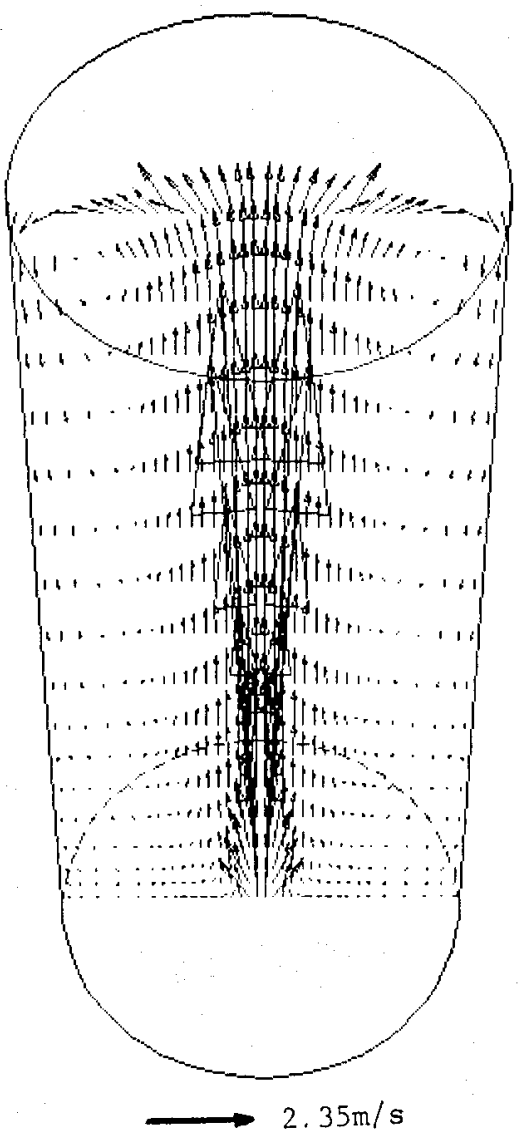

Fig. 8. Computed velocity vectors in a water model for the experimental conditions of Grevet et al..$^{5}$

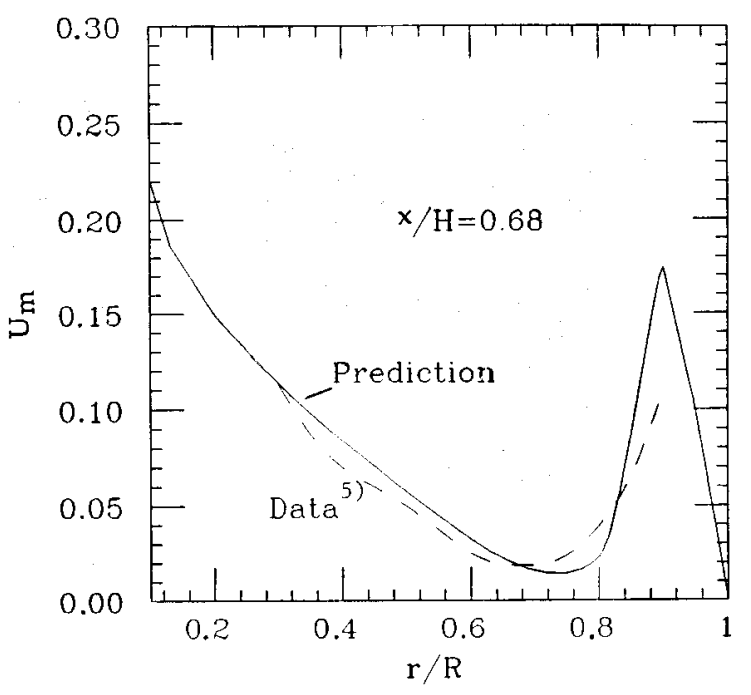

Fig. 9. Computed and measured magnitude of the mean axial velocity at $x / H=0.68$ for the experimental conditions of Grevet $e t$ al. ${ }^{5}$

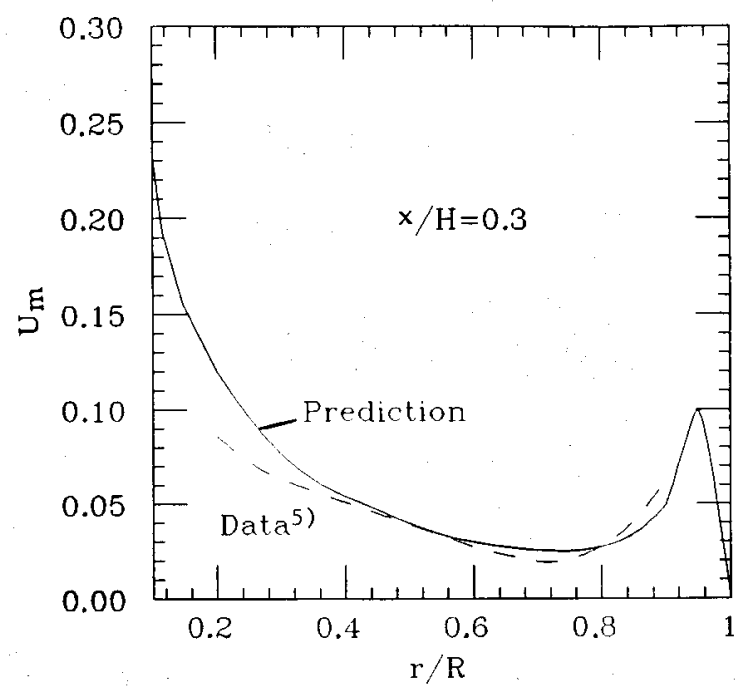

Fig. 10. Computed and measured magnitude of the mean axial velocity at $x / H=0.3$ for the experimental conditions of Grevet et al..$^{5}$

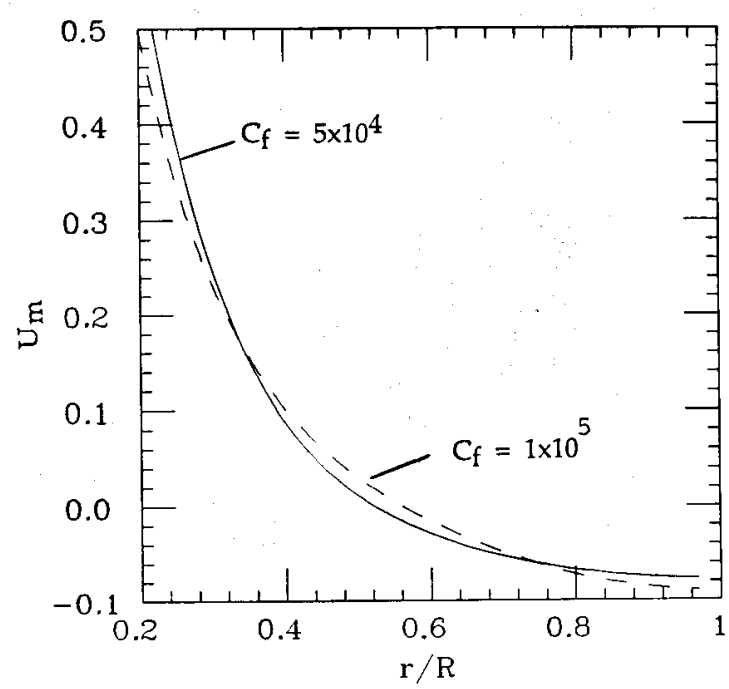

Fig. 11. Effect of friction coefficient $C_{f}$ on mean axial velocity at $x / H=0.1$ for the experimental conditions of Grevet $e t$ al..$^{5}$ 
agreement with the measurements, both regarding the void fraction distribution and the velocity profiles. The important point to be made here is that this agreement was not brought about by the use of any adjustable parameters to represent the plume shape.

\subsection{The Molten Steel System}

Ultimately the objective of modeling gas plumes in liquids has to be the representation of molten metal systems. This is quite a critical issue, because of the longstanding controversy whether the air-water systems can reliably represent molten metal-gas situations.

In the following we shall present selected calculations depicting the behavior of a steel-nitrogen system, the characteristic parameters of which are summarized in Table 4 . These are believed to correspond to typical steel processing conditions. Has argon been used instead of nitrogen, this would not have made any significant difference regarding the fluid flow behavior of the system.

Figs. 12 to 14 show the computed velocity profile and the void fraction distributions. A comparison of these plots with those previously given for an airwater system reveals quite significant differences. It is seen that for steel the plume is much narrower and consequently a much smaller volume of the vessel is being affected (i.e., agitated) by the presence of the gas plume. A very similar situation is being depicted in the gas fraction plots, that appear in Figs. 13 and 14. These clrearly show that a much higher gas fraction is being retained at the axis and as a corollary there is a small gas volume at distances away from the axis.

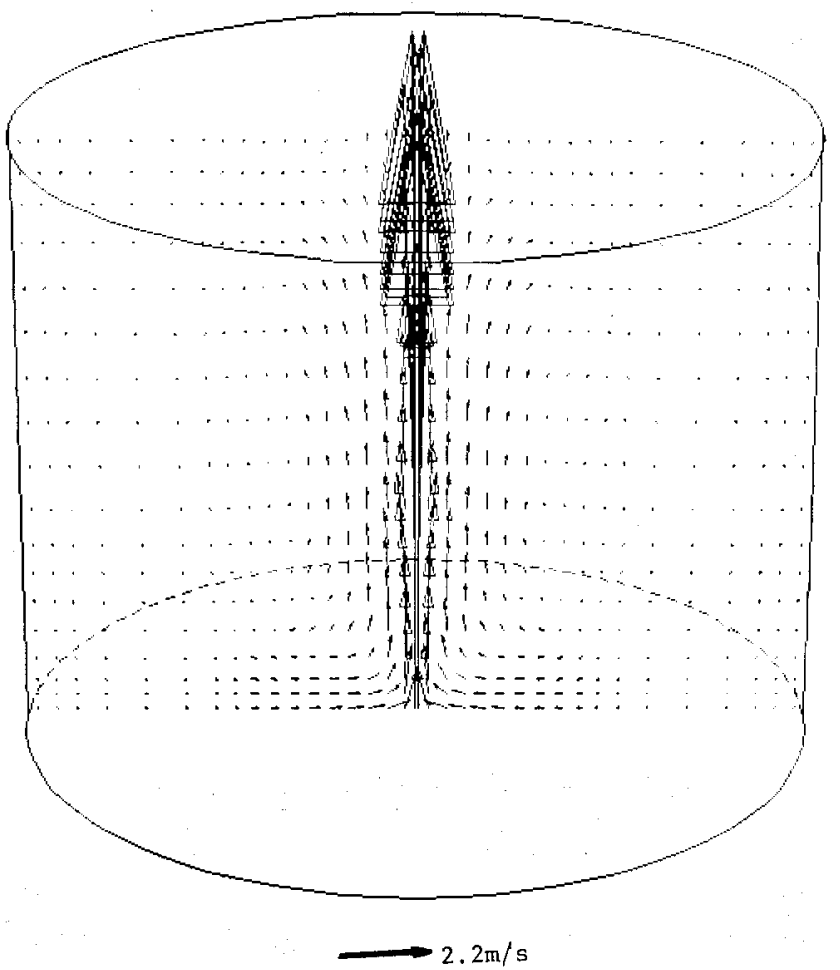

Fig. 12. Computed velocity vectors for a steel-nitrogen system.
There are many factors that may be responsible for this difference including,

-bubble diameter

-gas-injection condition

-effective viscosity.

In order to determine the relative contribution of each of the above factors, a systematic and more rigorous study than has been presented. Nonetheless, the present preliminary finding has very important potential practical consequences in that mixing rates and velocity fields that one might predict through the use of water models may not be fully applicable to molten metal systems.

\section{Discussion}

A mathematical representation has been proposed to describe the behavior of gas-plume driven circulation systems for both air in water and nitrogen in steel. The approach taken here was different from that usually employed in the modeling of such situa-

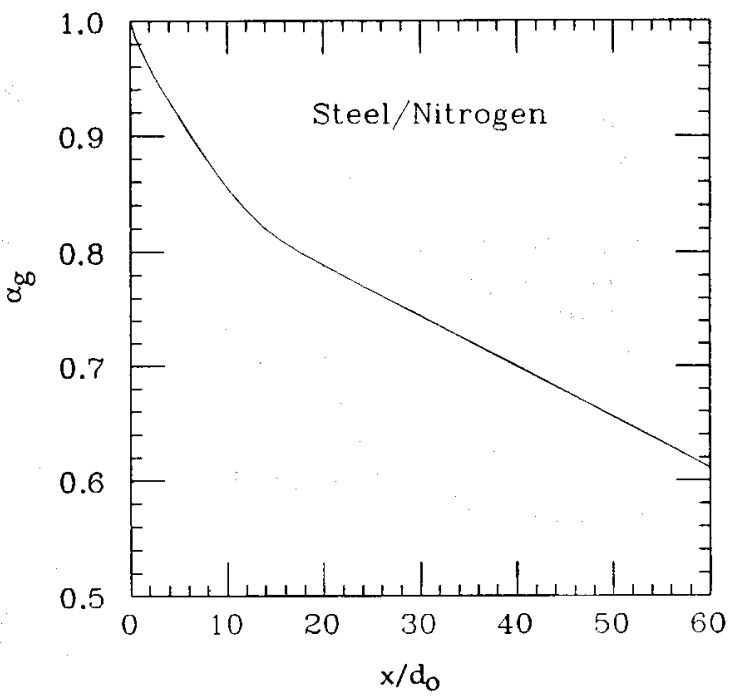

Fig. 13. Computcd axial variation of the gas fraction in a steel-nitrogen system.

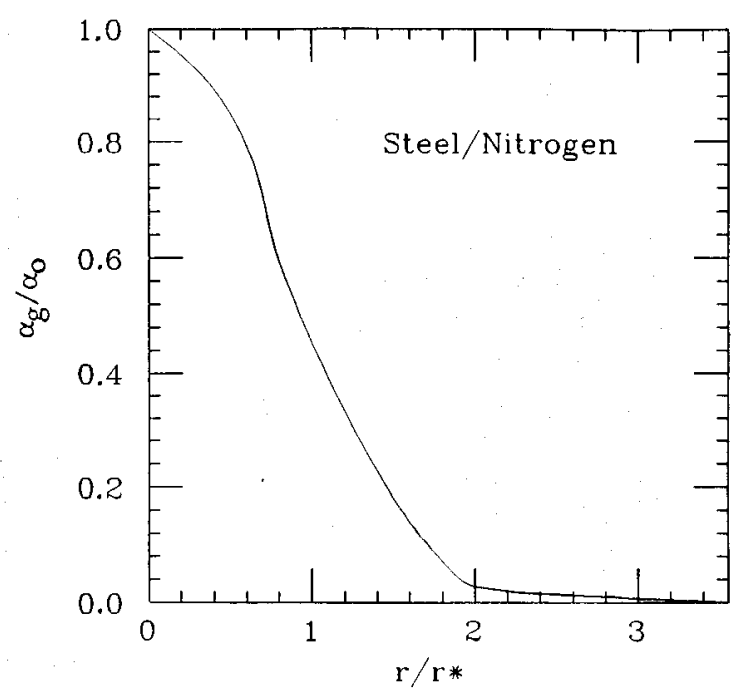

Fig. 14. Computed similarity profile of gas fraction in a steel-nitrogen system. 
tions, where the actual shape of the gas plume was postulated a priori. In the present work we represented the gas and the liquid as two separate phases and made due allowance for the interaction between them. This technique is an extension of prior work by Salcudean, ${ }^{97}$ Cross et al. ${ }^{10)}$ and Schwartz et al. ${ }^{11)}$

The approach outlined here is attractive because no arbitrary assumptions are needed regarding the plume shape and the only postulate required is to specify the bubble size and hence the interface-friction coefficient. Indeed sensitivity calculations described in the paper have shown that the mean flow results are not very sensitive to the bubble size chosen. Furthermore, the actual bubble size has been estimated using energy balance considerations.

The computed results for an air-water system were found to be in excellent agreement with the very detailed measurements of Castillejos and Brimacombe ${ }^{17)}$ regarding the void fraction distribution. Furthermore, the predicted liquid velocities agreed well with the previously published values of Grevet et al. $\left.{ }^{5}\right)$ This critical comparison of the predictions with measurements provides useful credibility for the model.

Calculations have also been performed to describe the behavior of nitrogen plumes in molten steel. These results were extremely interesting, because they suggest that gas plumes in molten metal behave quite differently from those in water. More specifically, the plumes appear to be much narrower and as a consequence the motion induced by them and the resultant agitation is significantly less than that found for the corresponding water systems.

This finding, which in view of the limited results, has to be regarded as somewhat tentative at this stage, would have very important implications. If correct, the results would suggest that water modeling studies could seriously overestimate the extent of mixing and agitation that can be produced by injected gas streams.

At present there is only a very limited experimental information that may be cited in support of this postulate. It should be noted, however, that in their paper, Schwartz et al. ${ }^{11)}$ found rather similar behavior, i.e., narrow gas plumes in iron baths compared to water.

It is suggested that the present work may open up a very interesting area of new inquiry. Experimental work would be highly desirable to test the results developed on the basis of the model. Further studies are currently in progress to study systematically the influence of bubble diameter on the flow parameters and a thorough investigation of the cffect of the additional terms employed in the transport equations.

Furthermore, the model could be made more realistic, through a proper allowance for the free surface disturbances caused by the gas bubbles as they cross the free surface and by representing the inherently unsteady nature of the gas plume. Work of this type is currently in progress in our laboratory.

\section{Nomenclature}

$C_{f}:$ Interface-friction coefficient $d_{b}$ : Bubble diameter

$d_{0}$ : Nozzle diamter

$D_{t}$ : Phase diffusion coefficient

$F_{u}$ : Interface friction per unit volume in $x$-direction

$F_{v}$ : Interface friction per unit volume in $y$-direction

$G$ : Generation rate of turbulence energy

$h, H$ : Height of liquid in vessel

$k$ : Turbulence kinetic energy

$P:$ Static pressure

$Q:$ Gas flow rate

$r$ : Radial coordinate

$r^{*}$ : Half-radius of plume (where $\alpha_{g}=0.5 \alpha_{0}$ )

$R:$ Radius of vessel

$R e_{b}:$ Reynolds number based on the bubble diameter

$S:$ Intra-fluid source terms

$S^{*}$ : Inter-fluid source terms

$U_{g}: \quad$ Gas velocity along $x$-direction

$U_{l}$ : Liquid velocity along $x$-direction

$U_{m}$ : Mean velocity

$V_{g}$ : Gas velocity along $r$-direction

$V_{l}:$ Liquid velocity along $r$-direction

$V r$ : Relative velocity between gas and liquid

$x$ : Axial coordinate direction

$\alpha_{g}:$ Gas fraction

$\alpha_{l}:$ Liquid fraction

$\alpha_{0}$ : Gas fraction at axis

$r:$ Interfacial tension

$\Gamma$ : Exchange coefficient

$\varepsilon$ : Rate of dissipation of turbulence energy

$\mu_{\mathrm{efr}}$ : Effective viscosity

$\mu_{l}:$ Laminar viscosity

$\mu_{t}$ : Turbulent viscosity

$\rho_{g}:$ Gas density

$\rho_{l}:$ Liquid density

$\phi$ : Generic fluid variable

\section{REFERENCES}

1) J. Szekely and K. Nakanishi: Iron Steel making, 3 (1975), 193.

2) J. Szekely and S. Asai: Trans. Iron Steel Inst. Jpn., 15 (1975), 276.

3) J. Szekely, H. J. Wang and K. M. Kisser: Metall. Trans. $B, 10 B$ (1976), 287.

4) T. Debroy, A. K. Majumdar and D. B. Spalding: J. Met., (1981), 42.

5) J. H. Grevet, J. Szekely and N. El-Kaddah: Int. J. Heat Mass Transfer, 25 (1982), 487.

6) J. W. Mckelliget, M. Cross and R. D. Gibson: Appl. Math. Modelling, 6 (1982), 469.

7) Y. Sahai and R.I.L. Guthrie: Metall. Trans. B, 13B (1982), 203.

8) M. Salcudean, C. H. Low, A. Hurda and R.I.L. Guthrie: Chem. Eng. Comm., 21 (1983), 89.

9) M. Salcudean, K.Y.M. Lai and R.I.L. Guthrie: Can. J. Chem. Eng., 63 (1984), 51.

10) M. Cross, N. C. Markatos and C. Aldham: Control '84: Proc. 1st Int. Symp. on Automatic Control in Minerals Processing and Process Metallurgy, J. A. Herbst, ed., AIMESME, New York, NY, (1984), 291-297.

11) M. P. Schwartz, J. K. Wright and B. R. Baldock: Proc. 
Symp. on Mathematical Modeling of Materials Processing Operations, J. Szekely et al., ed., Metall. Soc., Palm Springs, CA, (1987), 565-579.

12) J. S. Woo, J. Szekely, A. H. Castillejos and J. K. Brimacombe: "A study on the mathematical modeling of turbulent recirculating flows in gas-stirred ladles", Metall. Trans. B, (1989), in press.

13) I. Sawada and 'T. Ohashi: Tetsu-to-Hagané, 73 (1987), 699.

14) S. Taniguchi, A. Kikuchi, H. Matsunaga and N. Bessho: Trans. Iron Steel Inst. Jpn., 28 (1988), 262.

15) V. Sahajwalla, J. K. Brimacombe and M. E. Salcudean: SGANINJEGT V, Part II, 5th Int. Symp. on Ladle Metallurgy, Lulea, (1989), 103-144.

16) D. B. Spalding: Mathematics and Computers in Simulation, XIII, (1981) 267-276.

17) A. H. Castillejos and J. K. Brimacombe: SCANINJEGT IV, Part I, 4th Int. Conf. on Injection Metallurgy, Lulea, Pap. No. 16, (1986).
18) A. H. Castillejos: "A study of the fluid dynamic characteristics of turbulent gas-liquid bubble plumes", $\mathrm{PhD}$. Thesis, Dept. of Metallurgical Engineering, Univ. of British Columbia, Canada, (1986).

19) M. R. Malin: "Turbulence modeling for flow and heat transfer in jets, wakes and plumes", PhD Thesis, Univ. of London, UK, (1986).

20) O. Simonin: PhD Thesis, Polytechnic Institute of Grenoble, France, (1989).

21) R. Clift, J. R. Grace and M. E. Weber: Bubbles, Drops and Particles, Academic Press Inc., New York, NY, (1978).

22) M. Sano, K. Mori and Y. Fujiyama: Tetsu-to-Hagané, 65 (1979), 1140.

23) B. E. Launder and D. B. Spalding: Comp. Meth. Appl. Mech. Engg., 3 (1974), 269.

24) S. V. Patankar and D. B. Spalding: Int. J. Heat Mass Transfer, 15 (1972), 1787. 